\title{
Características clínicas y epidemiológicas de pacientes con tuberculosis en el estado Carabobo, Venezuela
}

\author{
Diomelis Maurera*, Gilberto Bastidas ${ }^{\dagger}$
}

\section{Resumen}

Introducción y objetivo: la tuberculosis (TB) es una de las principales causas de mortalidad a nivel mundial con tendencia al estancamiento en su control, situación a la que no es ajena Venezuela, y particularmente el estado Carabobo con importantes cifras de enfermedades respiratorias. El objetivo de este trabajo fue describir las características clínico-epidemiológicas de la TB ocurrida durante un año en el estado Carabobo.

Método: estudio descriptivo, de campo y retrospectivo.

Resultados: fueron en total 712 casos de TB pulmonar y 15 de TB extrapulmonar, mayoritariamente del municipio Valencia del estado Carabobo con afectación fundamental del sexo masculino (76\% y $67 \%$, respectivamente), además $67 \%$ de los afectados con la forma pulmonar se encuentran en edad económicamente productiva así como $53 \%$ de los pacientes con la variante extrapulmonar, siendo la meníngea la variante extrapulmonar de mayor frecuencia. Asimismo, 16,1\% de los pacientes con TB pulmonar tenían privación de libertad. Los afectados con TB tienen en mayor proporción como factor de riesgo la ingesta de alcohol y como patología sobreagregada la desnutrición. Los afectados con TB pulmonar muestran la sintomatología clásica con patrón radiológico bilateral cavitario de TB secundaria.

Conclusiones: la tasa de morbilidad por TB en 2017 muestra importante alza para el estado Carabobo, contraria a la tendencia mundial al descenso. Este trabajo se constituye en una puesta al día en la caracterización clínica-epidemiológica de la TB en el estado Carabobo con el objeto de aportar a los organismos oficiales información para la toma de decisiones sanitarias en el efectivo control de esta enfermedad.

\begin{tabular}{|c|c|c|c|}
\hline Palabras clave: & $\begin{array}{l}\text { Tuberculosis } \\
\text { Venezuela } \\
\text { Morbilidad } \\
\text { Epidemiología }\end{array}$ & Key words: & $\begin{array}{l}\text { Tuberculosis } \\
\text { Venezuela } \\
\text { Morbidity } \\
\text { Epidemiolog }\end{array}$ \\
\hline
\end{tabular}

\footnotetext{
* Doctora en Ciencias Sociales. Mención Salud y Sociedad. Docente e investigadora del Departamento de Salud Pública, Facultad de Ciencias de la Salud, Universidad de Carabobo. Facultad de Ciencias de la Salud, Universidad de Carabobo.

${ }^{\dagger}$ Doctor en Parasitología. Docente e investigador del Departamento de Salud Pública e Instituto de Investigaciones Médicas y Biotecnológicas,

Facultad de Ciencias de la Salud, Universidad de Carabobo, Venezuela.

Los autores declaran no tener conflictos de intereses.

Artículo aprobado en lo bioético por la Dirección de Programas de Salud de la Fundación Instituto Carabobeño para la Salud del estado

Carabobo, Venezuela,

Correspondencia: Urbanización Terrazas de San Diego, Sector C, Torre 14, Apartamento 14-54, municipio San Diego, estado Carabobo, Vene-

zuela. Correo electrónico: bastidasprotozoo@hotmail.com

Recibido: $17 / 10 / 18$

Aprobado: $11 / 3 / 19$
} 


\section{Introducción y antecedentes}

La tuberculosis, infección bacteriana causada por Mycobacterium tuberculosis, es una de las principales causas de mortalidad a nivel mundial con amplia variabilidad en la tasa de incidencia entre los países. En 2016, 10,4 millones de personas enfermaron y $1,7 \mathrm{mi}-$ llones murieron por esta patología, correspondiéndole a los países de bajos y medios ingresos económicos más de $95 \%$ de los fallecimientos. África tiene la mayor tasa de incidencia (incidencia media mundial de 133 casos por 100.000 habitantes) con más de 281 casos por 100.000 habitantes y son 22 los países donde se concentra alrededor de $80 \%$ de los casos, especialmente India, Indonesia, Nigeria, Pakistán, la República Popular de China y Sudáfrica ${ }^{(1-3)}$.

Ahora bien, atañe a los países con altos ingresos económicos, como los ubicados en el oeste de Europa, Canadá, Estados Unidos, Australia y Nueva Zelanda las tasas de incidencia más bajas, ya que reportan menos de 10 casos por 100.000 habitantes, y a los países del continente americano tasas inferiores a 50 por 100.000 habitantes, con 228.943 casos para el año 2017. La morbilidad está en relación directa con la pobreza, la malnutrición y las condiciones de hacinamiento e insalubridad, lo que hace que esta enfermedad sea considerada como un importante problema de salud pública en los países con bajos ingresos económicos ${ }^{(1-5)}$.

En Venezuela para el año 2010 se reportaron 6.213 nuevos casos de tuberculosis (TB), con tasas de prevalencia de 48 y de mortalidad de 3,1 casos por cada 100.000 habitantes, con tendencia al incremento de la casuística debido fundamentalmente al deterioro de las condiciones socioeconómicas, a la defíciencia en los programas de control y al descuido de la vigilancia epidemiológica, en este sentido, en el país, ya para la semana 52 del año 2014 se habrían producido 1.752 casos de TB y para la semana 26 del año 2015 (fecha hasta la cual se tienen registros oficiales) se alcanzaban los $1.598 \mathrm{ca}-$ sos, para el año 2016 se reportaron 7.816. Situación epidemiológica venezolana (tendencia al incremento de la tasa de infección) que pudiera estarse presentando en Carabobo, estado venezolano (registra 508 casos nuevos con una tasa de 20,6 por cada 100.000 habitantes para el año 2016) que posee importantes cifras de enfermedades del sistema respiratorio (representa 47,1\% del total de las causas de enfermedad del estado), pero sin que se muestre consistentemente la casuística de $\mathrm{TB}^{(6-9)}$.

La tendencia al estancamiento en el control de la TB puede verse favorecida por la pobreza creciente, la inmigración de países con elevada endemia tuberculosa, el descuido o abandono de los programas sanitarios de control, y al creciente número de individuos inmuno- comprometidos (se registran 1,4 millones de nuevos casos de TB en pacientes VIH positivos y en ellos representa la causa de una de cada tres defunciones), a estos factores se le suman el patrón clínico de enfermedad latente descrita en una importante proporción de la población mundial, el desarrollo de TB multirresistente (evento que se estima se produce en 480.000 personas a nivel mundial debido al fracaso en los programas de control, fallas en la supervisión e incumplimiento del tratamiento), y las reinfecciones exógenas como causa de recurrencia (debido a la simultánea coinfección con diferentes cepas o la reciente señalada compartamentalización de la infección con genotipos distintos que infectan diferentes tejidos) $)^{(7,10-14)}$.

La situación epidemiológica de la TB en relación con el incremento en el número total de casos, la carencia de información y la distribución heterogénea de la prevalencia (producto de la combinación de factores demográficos, como las migraciones y el hacinamiento; económicos, el desempleo y los bajos ingresos, y biológicos, la resistencia del microorganismo al tratamiento, la desnutrición, el tabaquismo y la infección por VIH) en estados como Carabobo con importantes áreas urbano marginales, donde puede resultar potenciada la transmisión de TB, hace necesaria la indagación de las características clínico-epidemiológicas, objetivo de la presente investigación, con el fin de ofrecer información sobre la magnitud del problema en estados venezolanos con Programa de Salud Respiratoria operativos, con el fin de proporcionar a los tomadores oficiales de decisión del estado Carabobo en particular y del venezolano en general información que garantice la correcta aplicación de los programas de control existentes y el rediseño de éstos en acuerdo con los factores de riesgo y vulnerabilidades identificadas.

\section{Material y método}

El presente es un estudio descriptivo con diseño de campo y retrospectivo basado previa aprobación bioética por la Dirección de Programas de Salud de la Fundación Instituto Carabobeño para la Salud (INSALUD), del estado Carabobo, Venezuela, en la revisión y confrontamiento estadístico de los datos contenidos en la ficha epidemiológica de TB y en el Informe Final de Tratamiento en relación con aspectos clínicos, demográficos y epidemiológicos que definen la TB (del Programa de Salud Respiratoria de INSALUD) en la región durante el año 2017. En el estudio se incluyeron los individuos de ambos sexos y todas las edades (los casos confirmados) en quienes se corroboró con pruebas microbiológicas, citológicas, histológicas o por imagen la patología. 
Tabla 1. Distribución proporcional de casos de TB pulmonar según sexo, edad y municipio. Estado Carabobo, Venezuela, 2017.

\begin{tabular}{|c|c|c|}
\hline \multicolumn{3}{|l|}{ Sexo* } \\
\hline Masculino & 541 & 76,0 \\
\hline Femenino & 171 & 24,0 \\
\hline \multicolumn{3}{|l|}{ Grupos de edad } \\
\hline 0 a 4 & 0 & 0,0 \\
\hline 5 a 14 & 4 & 0,6 \\
\hline 15 a 24 & 229 & 32,2 \\
\hline 25 a 34 & 251 & 35,3 \\
\hline 35 a 44 & 94 & 13,2 \\
\hline 45 a 54 & 71 & 10,0 \\
\hline 55 a 64 & 38 & 5,3 \\
\hline 65 y más & 25 & 3,5 \\
\hline \multicolumn{3}{|l|}{ Municipio** } \\
\hline Bejuma & 29 & 4,1 \\
\hline Carlos Arvelo & 37 & 5,2 \\
\hline Diego Ibarra & 26 & 3,7 \\
\hline Guacara & 25 & 3,5 \\
\hline Juan José Mora & 17 & 2,4 \\
\hline Libertador & 275 & 38,6 \\
\hline Los Guayos & 19 & 2,7 \\
\hline Miranda & 3 & 0,4 \\
\hline Montalbán & 0 & 0,0 \\
\hline Naguanagua & 22 & 3,1 \\
\hline San Diego & 8 & 1,1 \\
\hline Puerto Cabello & 52 & 7,3 \\
\hline San Joaquín & 14 & 2,0 \\
\hline Valencia & 185 & 26,0 \\
\hline
\end{tabular}

Fuente: Dirección de Programas de Salud del Instituto Carabobeño para la Salud (INSALUD).

$\chi^{2}: *$ Con diferencia estadísticamente significativa entre sexos $(\mathrm{p}<0,0000000)$.

**Con diferencia estadísticamente significativa entre el número de casos del municipio Libertador y Valencia $(\mathrm{p}<0,0000000)$.
La TB pulmonar para esta investigación se define, utilizando los criterios de clasificación de la Organización Mundial de la Salud (OMS), como aquella infección producida por Mycobacterium tuberculosis que afecta específicamente al parénquima pulmonar y la extrapulmonar aquella que afecta tejidos diferentes a éste ${ }^{(15)}$. Con la información obtenida se realizaron distribuciones de frecuencia y estadísticas descriptivas. Para probar la dependencia entre variables de interés se empleó la prueba $\chi^{2}$ y el coeficiente de correlación de Pearson ${ }^{(16)}$. Se aceptaron como significativas las pruebas con $\alpha \leq 0,05$.

\section{Resultados}

En total fueron 712 los casos de TB pulmonar reportados para el estado Carabobo en el año 2017, de éstos, $76 \%$ (541/712) ocurrieron en hombres y $24 \%$ (171/712) en mujeres (con diferencia estadística $\mathrm{p}<0,0000000$ ). Los grupos de edad más afectados fueron los ubicados entre 25 y 34 años $(35,3 \%)$ y entre 15 y 24 años $(32,2 \%)$ $(\mathrm{p}=0,15)$. El municipio del estado Carabobo con mayor reporte de TB pulmonar fue Libertador con 275 de los 712 casos $(38,6 \%)$, seguido por el municipio Valencia con $26 \%$ de los casos (con diferencias significativas entre ambos municipios $p<0,0000000)$. Asimismo, es importante señalar que de los 275 casos registrados en el municipio Libertador, 41,5\% (114/275) provienen del Complejo Penitenciario Carabobo y que en el municipio Montalbán no hubo casos de TB pulmonar (tabla 1).

La manifestación radiológica predominante fue la bilateral con cavitación (46,3\% [145/313]) seguida de la unilateral también con cavitación $(36,4 \%$ [114/313]) (con diferencias significativas entre ambas $[\mathrm{p}<0,005]$ ), patrón radiológico, el cavitario, característica de la TB secundaria. La principal patología concomitante fue la desnutrición (39,5\% [281/712]) con diferencia significativa con la diabetes mellitus ( $\mathrm{p}<0,0000000)$, la segunda en frecuencia (3,1\% [22/712]). La privación de liber$\operatorname{tad}(38,8$ [276/712]), el tabaquismo (25,8\% [184/712]) y la ingesta de alcohol (25,0\% [178/712]) son las condiciones de riesgo más frecuentes en la muestra estudiada (con diferencia significativa entre la privación de la libertad como condición de riesgo y las otras dos, $\mathrm{p}<0,0000000$ ) (tabla 2).

Las personas con TB extrapulmonar fueron 15 , del sexo masculino $67 \%(10 / 15)$ y del femenino $33 \%(5 / 15)$ ( $\sin$ diferencias significativas entre ambos sexos $(\mathrm{p}=$ $0,14)$. En este caso son tres los grupos de edad en quienes con mayor frecuencia se presenta la patología a saber: de 4 a 14 años (26,7\%), 15 a 24 años $(33,3 \%$ ) y de 35 a 44 años (33,3\%), pero sin diferencia significativa entre los grupos $(\mathrm{p}=0,63)$. La TB extrapulmonar es más frecuente en los municipios Valencia $(53,3 \%$ [8/15]) y Los 
Tabla 2. Distribución proporcional de casos de TB pulmonar según condición clínica, manifestación radiológica, comorbilidad y condición de riesgo. Estado Carabobo, Venezuela, 2017.

\begin{tabular}{|c|c|c|}
\hline Condición clínica & $f$ & $\%$ \\
\hline Asintomático & 0 & 0,0 \\
\hline Sintomático & 712 & 100,0 \\
\hline \multicolumn{3}{|l|}{ Manifestación radiológica* } \\
\hline Unilateral-infiltrado & 26 & 3,7 \\
\hline Unilateral-cavitación & 114 & 16,0 \\
\hline Bilateral cavitación & 145 & 20,4 \\
\hline Bilateral-infiltrado & 26 & 3,7 \\
\hline No practicado & 399 & 56,0 \\
\hline \multicolumn{3}{|l|}{ Comorbilidad $^{* *}$} \\
\hline Cáncer & 4 & 0,6 \\
\hline Desnutrición & 281 & 39,5 \\
\hline Diabetes mellitus & 22 & 3,1 \\
\hline Enfermedad renal & 3 & 0,4 \\
\hline $\mathrm{VIH} / \mathrm{SIDA}$ & 9 & 1,3 \\
\hline Ninguna & 393 & 55,2 \\
\hline \multicolumn{3}{|l|}{ Condición de riesgo*** } \\
\hline Consumo de sustancias psicotrópicas & 22 & 3,1 \\
\hline Exclusión social & 4 & 0,6 \\
\hline Ingesta de alcohol & 178 & 25,0 \\
\hline Privación de libertad & 276 & 38,8 \\
\hline Tabaquismo & 184 & 25,8 \\
\hline Ninguna & 210 & 29,5 \\
\hline \multicolumn{3}{|c|}{$\begin{array}{l}\text { Fuente: Dirección de Programas de Salud del Instituto Carabobe- } \\
\text { ño para la Salud (INSALUD). } \\
\chi^{2}:{ }^{*} \text { Con diferencia significativa entre los patrones radiológicos } \\
\text { unilateral con cavitación y bilateral con cavitación }(\mathrm{p}<0,005) \text {. } \\
\text { ** Con diferencia significativa entre desnutrición y diabetes me- } \\
1 \text { litus ( }<00,0000000 \text { ). } \\
\text { *** Con diferencia significativa entre privación de libertad y taba- } \\
\text { quismo e ingesta de alcohol ( } \mathrm{p}<0,0000000 \text { para cada caso). }\end{array}$} \\
\hline
\end{tabular}

Guayos (20,0\% [3/15]) (con diferencias significativas entre ambos municipios del estado Carabobo $(\mathrm{p}<0,05)$ (tabla 3).

La localización meníngea fue la más reportada con $16,7 \%(9 / 15)$, seguida por la ganglionar y la pericárdica, ambas con la misma proporción $(3,7 \%$ [2/15]) (con diferencias significativas entre cada una de estas y la gan- glionar $[\mathrm{p}<0,005])$. Respecto a las patologías que acompañan a los pacientes con TB extrapulmonar destacan con igual proporción la desnutrición y el VIH/SIDA. La ingesta de alcohol $(13,3 \%$ [2/15]) aparece de primero como factor de riesgo encontrado en pacientes con TB extrapulmonar, aunque sin diferencia significativa con el resto de factores también señalados $(\mathrm{p}=0,45)$ (tabla 4 ). En todos los casos se empleó el tratamiento acortado estrictamente supervisado (TAES) y las recaídas luego del tratamiento se presentaron en 3,6\% (20/541) hombres y $7,0 \%(12 / 171)$ mujeres con TB pulmonar. Únicamente un hombre y una mujer con TB pulmonar resultaron multidrogorresistentes. En el caso de TB extrapulmonar no se registró resistencia a fármacos, ni tampoco recaídas.

\section{Discusión}

$\mathrm{Al}$ igual que en otros estudios y aéreas geográficas los hombres fueron los más afectados por TB (enfermedad que encierra discriminación y estigmatización para el afectado por sus iguales sanos) posiblemente porque en ellos se registran con mayor frecuencias los factores de riesgo (tabaquismo, alcoholismo, malnutrición y marginalidad), y los grupos de edad mayormente afectados por la TB son los considerados económicamente productivos, sin que esto necesariamente implique que los demás grupos, particularmente los ubicados en los extremos de la vida, resulten particularmente exentos de riesgo ${ }^{(2,17)}$. La desigualdad de sexo (mayor cantidad de casos en el masculino) en cuanto al padecimiento de TB marca a la población estudiada, como sucede en la mayoría de los países en que esta patología es común, de la misma manera la brecha es más importante en el grupo poblacional considerado el motor de la economía, es decir, entre quienes tienen entre 20 y 34 años ${ }^{(17)}$.

Ahora bien, las mayores prevalencias de TB en cuanto al área geográfica afectada en el estado Carabobo están directamente relacionadas con condiciones de pobreza y hacinamiento, pues el municipio Valencia tiene la mayor población del estado y proporción de zonas urbanas marginales y rurales económicamente deprimidas; además, al municipio Libertador se le agrega el asentamiento en su territorio del mayor y más importante centro penitenciario del estado, donde el hacinamiento, las fallas de infraestructura y servicios de habitabilidad básicos y sanitarios son los elementos claves que lo caracterizan. Véase que lo hallado para TB en el estado Carabobo se mantiene dentro de la gran desigualdad, ya descrita, en la distribución de los casos entre los países y hacia el interior de cada uno de ellos, de allí deriva la importancia de reportes como el realizado con este escrito para el control de esta patología infecciosa ${ }^{(2,17-19)}$. 
Tabla 3. Distribución proporcional de casos de TB extrapulmonar según sexo, edad y municipio. Estado Carabobo, Venezuela, 2017.

\begin{tabular}{|c|c|c|}
\hline \multicolumn{3}{|l|}{ Sexo } \\
\hline Masculino & 10 & 67,0 \\
\hline Femenino & 5 & 3,0 \\
\hline \multicolumn{3}{|l|}{ Grupos de edad } \\
\hline 0 a 4 & 0 & 0,0 \\
\hline 5 a 14 & 4 & 26,7 \\
\hline 15 a 24 & 5 & 33,3 \\
\hline 25 a 34 & 1 & 6,7 \\
\hline 35 a 44 & 5 & 33,3 \\
\hline 45 a 54 & 0 & 0,0 \\
\hline 55 a 64 & 0 & 0,0 \\
\hline 65 y mas & 0 & 0,0 \\
\hline \multicolumn{3}{|l|}{ Municipio** } \\
\hline Bejuma & 0 & 0,0 \\
\hline Carlos Arvelo & 1 & 6,7 \\
\hline Diego Ibarra & 0 & 0,0 \\
\hline Guacara & 0 & 0,0 \\
\hline Juan José Mora & 0 & 0,0 \\
\hline Libertador & 1 & 6,7 \\
\hline Los Guayos & 3 & 20,0 \\
\hline Miranda & 0 & 0,0 \\
\hline Montalbán & 0 & 0,0 \\
\hline Naguanagua & 1 & 6,7 \\
\hline San Diego & 0 & 0,0 \\
\hline Puerto Cabello & 1 & 6,7 \\
\hline San Joaquín & 0 & 0,0 \\
\hline Valencia & 8 & 53,3 \\
\hline $\begin{array}{l}\text { Fuente: Dirección } \\
\text { ño para la Salud (I } \\
\chi^{2}:{ }^{*} \text { Con diferenc } \\
\text { de casos del munic }\end{array}$ & de & $\begin{array}{l}\text { Carabobe- } \\
\text { e el número } \\
\text { 5). }\end{array}$ \\
\hline
\end{tabular}

Lo aquí hallado (una prevalencia de 16,01\% [114/712] de TB pulmonar en privados de libertad) encaja perfectamente con el hecho, reportado en investigaciones previas, que la TB es la primera causa de mortalidad en la población que resulta privada de libertad, a tal punto que se estima que pueden producirse como tasa de incidencia hasta 7.000 casos de esta patología por cada 100.000 recluidos en centros penitenciarios por año, lo que constituye a la luz de otros grupos poblacionales una muy alta incidencia, que a la larga ayuda con el aumento del riesgo para contagio de TB en la población general donde se encuentra instalado el centro de reclusión penitenciaria, porque los estudios muestran que cerca de $6,3 \%$ de los infecciones con el bacilo ácido alcohol resistente de la TB se deben al contacto con la población privada de libertad ${ }^{(20-25)}$. Entonces, en lo que respecta al estado Carabobo, puede afirmarse que la situación ha cambiado poco en relación con el grave problema de salud pública en que se han constituido los centros penitenciarios, particularmente los de países en vías de desarro$110^{(26)}$.

La tos productiva (a veces con sangre en el esputo), dolor torácico, debilidad, pérdida de peso, y fiebre y sudoración nocturna, con ligeras diferencias en cuanto a la cantidad e intensidad de los mismos, fueron los síntomas comunes en los pacientes con TB pulmonar de la población carabobeña estudiada, no hubo entonces cuadros atípicos en cuanto a manifestaciones clínicas se refie$\mathrm{re}^{(2,27)}$. Asimismo, el diagnóstico radiológico mostró mayoritariamente los patrones frecuentes de TB pulmonar (infiltrado y cavitario); no obstante, el patrón radiológico predominante fue el bilateral cavitario característico de la TB secundaria, este hallazgo pone en evidencia la efectividad del programa de salud respiratoria en el estado Carabobo, en término de prevención de la enfermedad, diagnóstico y tratamiento ${ }^{(28)}$.

Los principales factores de riesgo para la adquisición de TB encontrados en este estudio fueron la privación de libertad (marcada en el estado Carabobo por hacinamiento y deficientes condiciones de salubridad), el tabaquismo y la ingesta de alcohol. En este sentido se conoce que el consumo de tabaco incrementa de forma importante el riesgo de enfermar de TB entre quienes tienen este hábito, y en algunos casos de morir por esta causa, es así que a nivel mundial se atribuye $8 \%$ de los casos de TB al tabaquismo ${ }^{(2)}$. Ahora bien, se imputa a la alteración en el equilibrio de citocinas del sistema inmunitario que el abuso del consumo de alcohol produce el mayor riesgo de padecer de TB observado en esta y otras poblaciones estudiadas a nivel mundial. Igualmente la desnutrición y la diabetes mellitus se constituyen en las principales patologías concomitantes observadas en los estudiados, esto en el mismo tenor con lo reportado con anterioridad; no obstante, el VIH como patología concomitante no tiene en este estudio la cuota de participación que habitualmente se le atribuye, posiblemente debido a la falta de diagnóstico o subregistro ${ }^{(17,29)}$. 
Tabla 4. Distribución proporcional de casos de TB extrapulmonar según órgano afectado, comorbilidad y condición de riesgo. Estado Carabobo, Venezuela, 2017.

\begin{tabular}{|c|c|c|}
\hline Características & $f$ & $\%$ \\
\hline \multicolumn{3}{|l|}{ Órgano afectado* } \\
\hline Ganglionar & 2 & 3,7 \\
\hline Meníngea & 9 & 16,7 \\
\hline Ósea & 1 & 1,9 \\
\hline Pericárdica & 2 & 3,7 \\
\hline Vulvar & 1 & 1,9 \\
\hline \multicolumn{3}{|l|}{ Comorbilidad } \\
\hline Cáncer & 0 & 0,0 \\
\hline Desnutrición & 2 & 13,3 \\
\hline Diabetes mellitus & 1 & 6,7 \\
\hline Enfermedad renal & 0 & 0,0 \\
\hline VIH/SIDA & 2 & 13,3 \\
\hline Ninguna & 10 & 66,7 \\
\hline \multicolumn{3}{|l|}{ Condición de riesgo } \\
\hline Consumo de sustancias psicotrópicas & 1 & 6,7 \\
\hline Exclusión social & 0 & 0,0 \\
\hline Ingesta de alcohol & 2 & 13,3 \\
\hline Personal de salud & 1 & 6,7 \\
\hline Privación de libertad & 1 & 6,7 \\
\hline Tabaquismo & 1 & 6,7 \\
\hline Ninguno & 9 & 60,0 \\
\hline \multicolumn{3}{|c|}{$\begin{array}{l}\text { Fuente: Dirección de Programas de Salud del Instituto Carabobe- } \\
\text { ño para la Salud (INSALUD). } \\
\chi^{2}: \text { *Con diferencia estadísticamente significativa entre la forma } \\
\text { de TB extapulmonar meníngea y pericárdica y ganglionar } \\
(p<0,005) \text {. }\end{array}$} \\
\hline
\end{tabular}

Al igual que la TB pulmonar los más afectados con TB extrapulmonar son los hombres en edad económicamente productiva y provenientes del municipio Valencia (municipio con mayor densidad de población del estado Carabobo). La TB extrapulmonar predominante en este estudio fue la del sistema nervioso central, específicamente la meníngea (dentro de este grupo es la forma más frecuente y tiene un curso clínico subagudo e insidioso) como consecuencia de la diseminación hemató- gena distal o del transcurso de una TB diseminada, aspecto (foco de origen) no descrito en el registro clínico. Contrario este hallazgo a la siempre mayor frecuencia señalada para la TB extrapulmonar ganglionar sobre cualquiera de las otras formas, con proporciones que oscilan entre $30 \%$ y $40 \%{ }^{(30,31)}$.

En todo caso la inmunosupresión por desnutrición, VIH/SIDA e ingesta de alcohol parece tener un importante papel en el desarrollo de la TB extrapulmonar meníngea en el grupo de pacientes revisados, en acuerdo con lo señalado en la literatura en relación con el elevado riesgo de TB extrapulmonar en personas con trastornos que dañan el sistema inmunitario, probabilidad que resulta entre 20 y 30 veces mayor en personas infectadas con $\mathrm{VIH}^{(2,31)}$. La resistencia a medicamento contra la TB fue prácticamente insignificante en los registros clínicos de los pacientes revisados y a expensas exclusivamente de la localización pulmonar de la enfermedad, esto a pesar que la TB multirresistente constituye actualmente un problema de salud pública, porque se estima, por ejemplo, que se producen 600.000 nuevos casos de resistencia a la rifampicina (fármaco de primera línea y más eficaz), 490.000 de los cuales son multirresistentes ${ }^{(2)}$.

La tendencia al incremento de la morbilidad por TB reportada para el estado Carabobo en el año estudiado se debe, sin duda, a pesar de la disposición del Programa de Salud Respiratoria (que proporciona métodos diagnósticos y tratamiento), a múltiples razones entre las que destacan los factores biológicos y la desnutrición, ambos relacionados con la vulnerabilidad del organismo a la infección y enfermedad (por deficientes condiciones de vida). En este sentido, las desfavorables condiciones sociales permite a los casos de TB permanecer por un tiempo variables antes de ser diagnosticados y tratados correctamente, lapso en que los infectados se constituyen en fuentes de diseminación de la enfermedad, donde las personas sanas que comparten mayor tiempo con los enfermos no detectados tienen mayor riesgo de infectarse y enfermar, entre ellos, las personas privadas de libertad ${ }^{(32-37)}$.

\section{Conclusiones}

Por último, se concluye que se produjo un importante incremento en la morbilidad por TB en el estado Carabobo en relación con la data registrada en años anteriores a 2017 y en contra a la tendencia mundial al descenso; que los hombres en edades económicamente productiva y provenientes del municipio Valencia del estado Carabobo, Venezuela, son los más afectados por TB; que los privados de libertad representan $16,1 \%$ del total de pacientes con TB pulmonar; que los afectados con TB pulmonar muestran la sintomatología clásica con patrón radiológico bilateral cavitario característico de la TB se- 
cundaria; que el tabaquismo y la ingesta de alcohol son los factores de riesgo de TB pulmonar mayormente encontrados con la desnutrición y diabetes mellitus como las principales patologías concomitantes; que la TB extrapulmonar meníngea fue la más frecuente y se presentó principalmente en hombres en edad productiva y del municipio Valencia, con VIH/SIDA y la ingesta de alcohol como los más importantes factores de riesgo; que este trabajo se constituye en una puesta al día de la caracterización clínica-epidemiológica de la TB en el estado Carabobo.

\section{Abstract}

Introduction: tuberculosis (TB) is one of the main causes of mortality worldwide with a tendency to stagnation in its control. Venezuela follows the global tendency, and in particular the state of Carabobo, which accounts for significant numbers of respiratory diseases. Objective: to describe the clinical-epidemiological characteristics of TB that occurred during one year in the state of Carabobo.

Method: descriptive, field and retrospective study.

Results: A total of 712 cases of pulmonary TB and 15 cases of extrapulmonary TB were part of the study, most of them in the municipality of Valencia of the state of Carabobo, with fundamental involvement of the male gender ( $76 \%$ and $67 \%$ respectively), and $67 \%$ of the patients affected being economically productive, as well as $53 \%$ of the patients with the extrapulmonary variant, being the meningeal the extrapulmonary variant of greater frequency. Likewise, $16.1 \%$ of patients with pulmonary TB were deprived of their liberty. Those affected with TB have a higher risk of alcohol intake as a risk factor and malnutrition as an added pathology. Those affected with pulmonary TB show the classic symptomatology with a bilateral cavitary radiological pattern of secondary TB.

Conclusions: the TB morbidity rate in 2017 shows an important increase for the Carabobo state, contrary to the global tendency to decrease, and this study constitutes an update on the clinical-epidemiological characterization of TB in the state of Carabobo with the purpose of providing official organizations with information for making health decisions for the effective control of this disease.

\section{Resumo}

Introdução e objetivo: a tuberculose (TB) é uma das principais causas de mortalidade no mundo, com tendência à estagnação no seu controle, uma situação que não é alheia na Venezuela, e particularmente no estado de Carabobo com um número significativo de doenças respiratórias. O objetivo deste trabalho é des- crever as características clínico-epidemiológicas da TB ocorridas durante um ano no estado de Carabobo.

Método: estudo descritivo, de campo e retrospectivo.

Resultados: foram registrados 712 casos de tuberculose pulmonar e 15 extrapulmonar principalmente no município de Valencia em Carabobo com maior incidência em pacientes do sexo masculino (76\% e $67 \%$, respectivamente); mais de $67 \%$ dos pacientes com a forma pulmonar estavam em idades economicamente produtivas, bem como $53 \%$ dos pacientes com a variante extrapulmonar, sendo a variante meníngea extrapulmonar a de maior frequência. $16,1 \%$ dos pacientes com TB pulmonar estavam privados de liberdade. Uma maior proporção dos pacientes afetados pela tuberculose apresenta como fator de risco a ingestão de álcool e de desnutrição como patologia adicional. Os acometidos por TB pulmonar apresentam a sintomatologia clássica com padrão radiológico cavitário bilateral de TB secundária.

Conclusões: a taxa de morbidade por TB em 2017 mostrou um aumento relevante no estado de Carabobo, oposta a tendência mundial de redução; este trabalho apresenta uma atualização sobre as características clínicas e epidemiológicas da tuberculose no estado de Carabobo com o objetivo de fornecer às organizações oficiais informação para a tomada de decisões sanitárias para o controle efetivo dessa doença.

\section{Bibliografía}

1. Organización Mundial de la Salud. Definiciones y marco de trabajo para la notificación de tuberculosis-revisión 2013 (actualizado en diciembre de 2014). Ginebra: OMS, 2013. Disponible en: http://apps.who.int/iris/bitstream/handle/ 10665/111016/9789243505343_spa.pdf;jsessionid= 8F28BD64669B473B8BF818BBA1E55EDC? sequence=1. [Consulta: noviembre 2017].

2. Organización Mundial de la Salud. Informe mundial sobre la tuberculosis. Sinopsis. OMS, 2017. Disponible en: http://www.who.int/tb/publications/global_report/gtbr2017 executive_summary_es.pdf?ua=1. [Consulta: marzo 2018].

3. Bojovic O, Medenica M, Zivkovic D, Rakocevic B, Trajkovic G, Kisic-Tepavcevic D, et al. Factors associated with patient and health system delays in diagnosis and treatment of tuberculosis in Montenegro, 2015-2016. PLoS One 2018; 13(3):e0193997. http://doi:10.1371/journal.pone.0193997.

4. Organización Panamericana de la Salud. Plan Regional de Tuberculosis 2006 - 2015. Washington, D.C.: OPS, 2006. Disponible en: http://www.bvsde.paho.org/texcom/cd045364/ TbRegPlan2006.pdf. [Consulta: enero 2018].

5. Organización Panamericana de la Salud, Organización Mundial de la Salud. Tuberculosis en las Américas 2018. Washington, D.C.: OPS, 2018. Disponible en: http://iris. paho.org/xmlui/bitstream/handle/123456789/49510/ 
OPSCDE18036_spa? sequence $=2 \&$ isAllowed $=y$. [Consulta: febrero 2019].

6. Piñate F, Avilán J. Estado actual de la lucha antituberculosa en Venezuela. Gac Méd Caracas 2007; 115(4):325-34. Disponible en: http://www.scielo.org.ve/pdf/s/v15n3/art10.pdf. [Consulta: noviembre 2017].

7. Méndez M. La tuberculosis pulmonar: pasado, presente y futuro en Venezuela. Salus 2011; 15(3):37-41. Disponible en: http://www.scielo.org.ve/scielo.php?script=sci_arttext\&pid= S1316-71382011000300010. [Consulta: marzo 2018].

8. Venezuela. Ministerio del Poder Popular para la Salud. Anuario de Morbilidad. Caracas, 2013. Disponible en: http://www.mpps.gob.ve. [Consulta: febrero 2018].

9. Venezuela. Ministerio del Poder Popular para la Salud. Boletín Epidemiológico. Semana epidemiológica $n^{\circ} 26$. Caracas, 2015. Disponible en: http://www.mpps.gob.ve. [Consulta: febrero 2018].

10. Arráiz N, Bermúdez V, Urdaneta B. Resistencia a drogas en M. tuberculosis: bases moleculares. AVFT 2005; 24:23-31. Disponible en: http://www.scielo.org.ve/scielo.php?script=sci_arttext\&pid=S0798-02642005000100004. [Consulta: abril 2018].

11. Comas I, Gagneux S. The past and future of tuberculosis research. PLoS Pathog 2009; 5: e1000600. Disponible: https://journals.plos.org/plospathogens/article?id=10.1371/ journal.ppat.1000600. [Consulta: junio 2018].

12. Prasad R. Multidrug and extensively drug-resistant TB (M/ XDR-TB): problems and solutions. Indian J Tuberc 2010; 57:180-91. Disponible en: http://medind.nic.in/ ibr/t10/i4/ibrt10i4p180.pdf. [Consulta: julio 2018].

13. Organización Panamericana de la Salud, Organización Mundial de la Salud. La tuberculosis en las Américas. Informe regional 2013: epidemiología, control y financiamiento. Washington, D.C., 2014. Disponible en: https://www. paho.org/hq/dmdocuments/2014/2014-cha-informeregional-tb.pdf. [Consulta: julio 2018].

14. Navarro Y, Romero B, Copano M, Bouza E, Domínguez L, de Juan L, et al. El muestreo múltiple y la huella digital discriminatoria revelan infecciones clonalmente complejas y compartimentadas por M. bovis en el ganado. Vet Microbiol 2015; 175(1):99-104.

15. World Health Organization. Global tuberculosis control: epidemiology, strategy, financing. Geneva: WHO, 2009.

16. Arias F. El proyecto de investigación: introducción a la metodología científica. Caracas: Episteme, 2004.

17. Andreu J, Jaén C, Segarra L, Flores J, Jaén R, Cerdá E. Asociación de tuberculosis y alcohol en pacientes de una unidad de infecciosos. Adicciones 2002; 14(1):9-11. Disponible en: https://www.adicciones.es/index.php/adicciones/article/download/508/503. [Consulta: agosto 2018].

18. Bossio C, Arias J, Fernández Hr. Tuberculosis en Argentina: desigualdad social y género. Salud Colectiva 2012; 8(Supl 1):S77-91. Disponible en: http://www.unla.edu.ar/saludcolectiva/revista23_bis/v8s1a13.pdf. [Consulta: marzo 2018].
19. Lonnroth K, Castro K, Chakaya J, Chauhan L, Floyd K, Glaziou P, et al. Tuberculosis control and elimination 2010-50: cure, care, and social development. Lancet 2010; 375(9728):1814-29. http://doi:10.1016/S0140-6736(10)60483-7.

20. Castañeda-Hernández D, Martínez-Ramírez J, Bolivar-Mejía A, Rodríguez-Morales A. Differences in TB incidence between prison and general populations, Pereira, Colombia, 2010-2011. Tuberculosis (Edinb) 2013; 93(3):275-6. http://doi:10.1016/j.tube.2013.02.001.

21. Rueda Z, Arroyave L, Marin D, López L, Keynan Y, Giraldo MR, et al. High prevalence and risk factors associated with latent tuberculous infection in two Colombian prisons. Int J Tuberc Lung Dis 2014; 18(10):1166-71. http://doi: 10.5588/ijtld.14.0179.

22. Valença M, Scaini J, Abileira F, Gonçalves C, von Groll A, Silva P. Prevalence of tuberculosis in prisons: risk factors and molecular epidemiology. Int J Tuberc Lung Dis 2015; 19(10):1182-7. http://doi:10.5588/ijtld.15.0126.

23. Banu S, Rahman M, Uddin M, Khatun R, Khan M, Rahman M, et al. Effect of active case finding on prevalence and transmission of pulmonary tuberculosis in Dhaka Central Jail, Bangladesh. PLoS One 2015; 10(5):e0124976. http://doi:10.1371/journal.pone.0124976.

24. Dara M, Acosta C, Melchers N, Al-Darraji H, Chorgoliani D, Reyes H, et al. Tuberculosis control in prisons: current situation and research gaps. Int J Infect Dis 2015; 32:111-7. http://doi:10.1016/j.ijid.2014.12.029.

25. Rodríguez L, Chimoy C. Características epidemiológicas de tuberculosis pulmonar en establecimiento penitenciario de varones de Trujillo-Perú. Acta méd Perú 2017; 34(3):182-7. Disponible en: http://www.scielo.org.pe/scielo.php?script= sci_arttext\&pid=S1728-59172017000300004. [Consulta: abril 2018].

26. Organización Panamericana de la Salud. Guía para el control de la tuberculosis en poblaciones privadas de libertad de América Latina y el Caribe: abordaje a poblaciones vulnerables. Washington, D.C.: OPS, 2008. Disponible en: http:// www1.paho.org/spanish/ad/dpc/cd/tbprisiones-guia-ctl.htm. [Consulta: marzo 2018].

27. Carvalho A, Cardoso C, Martire T, Migliori G, Sant'Anna C. Epidemiological aspects, clinical manifestations, and prevention of pediatric tuberculosis from the perspective of the End TB Strategy. J Bras Pneumol 2018; 44(2):134-44. http:// doi: 10.1590/S1806-37562017000000461

28. Serrano F, Moreno I. Manifestaciones radiológicas de la tuberculosis. Arch Bronconeumol 1983; 19(6):248-54. Disponible en: https://medes.com/publication/103110. [Consulta: agosto 2018].

29. Cook R. Alcohol abuse, alcoholism, and damage to the immune system- a review. Alcohol Clin Exp Res 1998; 22(9):1927-42. https://doi.org/10.1111/j.1530-0277.1998.tb05900.x.

30. Peto H, Pratt R, Harrington T, LoBue P, Armastrong L. Epidemiology of extrapulmonary tuberculosis in the United States, 1993-2006. Clin infect Dis 2009; 49:1350-7. http://doi:10.1086/605559. 
31. Ramírez-Lapausa M, Menéndez-Saldaña A, Noguerado-Asensio A. Tuberculosis extrapulmonar, una revisión. Rev Esp Sanid Penit 2015; 17: 3-11. Disponible en: $\mathrm{http} / / /$ scielo.isciii.es/pdf/sanipe/v17n1/02_revision.pdf. [Consulta: agosto 2018].

32. Organización Mundial de la Salud. Plan mundial para detener la tuberculosis 2006-2015/ Alianza Alto a la Tuberculosis. Ginebra: OMS, 2006. Disponible en: http://www.who.int/mediacentre/news/releases/2007/pr25/es/. [Consulta: junio 2018].

33. Young D, Perkins M, Duncan K, Barry C. Confronting the scientific obstacles to global control of tuberculosis. J Clin Invest 2008; 118:1255-65. Disponible en: https:// www.ncbi.nlm.nih.gov/pmc/articles/PMC2276805/. [Consulta: septiembre 2018].

34. Lonnroth K, Jaramillo E, Williams B, Dye C, Raviglione M. Drivers of tuberculosis epidemics: the role of risk factors and social determinants. Soc Sci Med 2009; 68(12):2240-6. http://doi:10.1016/j.socscimed.2009.03.041.

35. Hargreaves J, Boccia D, Evans C, Adato M, Petticrew M, Porter J. The social determinants of tuberculosis: from evidence to action. Am J Public Health 2011; 101(4):654-62. Disponible en: https://www.ncbi.nlm.nih.gov/pmc/articles/PMC3052350/. [Consulta: junio 2018].

36. Creswell J, Jaramillo E, Lonnroth K, Weil D, Raviglione M. Tuberculosis and poverty: what is being done. Int J Tuberc Lung Dis 2011; 15(4):431-2. http://doi: 10.5588/ijtld.10.0654.

37. Yagui M, Jave H, Curisinche M, Gutiérrez C, Romaní F. Agenda Nacional de Investigación en Tuberculosis en Perú, 2011-2014. Rev Panam Salud Pública 2013; 33(2):151-8. Disponible en https://www.scielosp.org/article/ssm/content/raw/?resource_ssm_path=/media/assets/rpsp/v33n2/11.pdf. [Consulta: junio 2018]. 